Interview

\section{Candesartan als sinnvolle Erweiterung der Herzinsuffizienztherapie}

Interview mit Prof. Dr. med. Bernd Krönig, Chefarzt der Abteilung Innere Medizin, Evang. Elisabeth-Krankenhaus, Trier

NOTFALL \& HAUSARZTMEDIZIN 2004; 30: B 274-B 275

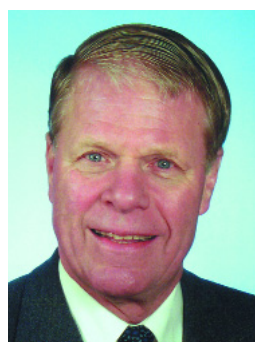

B. Krönig
Frage: Herr Prof. Krönig, welches Ergebnis der CHARM-Studie hat Sie am meisten beeindruckt?

Prof. Krönig: Die CHARM-Studie ist eine besonders herausragende Studie, weil sie an einem großen Kollektiv von über 7600 Patienten mit mehr oder minder fortgeschrittener Herzinsuffizienz durchgeführt worden ist und mit einer bisher noch wenig eingesetzten Kombination von ACEHemmer und $\mathrm{AT}_{1}$-Rezeptorblocker einen zusätzlichen Benefit gebracht hat. Jeder in Klinikum und Praxis weiß, dass Patienten mit schwerer Herzinsuffizienz - sei es postischämisch oder im Rahmen einer hypertensiven Herzkrankheit - ein schwer zu therapierendes Klientel sind. Alle drei Studien des CHARM-Programms - $\mathrm{CH}$ ARM-Added, CHARM-Alternative und CHARM-Preserved - haben gezeigt, dass die Gabe eines $\mathrm{AT}_{1}$-Rezeptorblockers, hier Candesartan, zusätzliche Vorteile für unsere Patienten bringt.

Besonders beeindruckend sind freilich die Ergebnisse von CHARMAdded bei einem Patientenkollektiv, was eine von vornherein schlechte linksventrikuläre Funktion mit einer Ejektionsfraktion von 28\% hatte und bereits auf Diuretika/Spironolacton, Betablocker und ACE-Hemmer eingestellt war. Auch bei dieser Patientengruppe brachte die zusätzliche Gabe des $\mathrm{AT}_{1}$-Rezeptorblockers eine signifikante Verbesserung.
Es gibt aber auch noch einige spezielle Punkte, da nicht nur die gesamte kardiovaskuläre Mortalität, sondern auch die Häufigkeit stationärer Krankenhauseinweisungen deutlich reduziert wurden. Gerade in Zeiten knapper Mittel im Gesundheitswesen stellt die Vermeidung einer - leider bei herzinsuffizienten Patienten häufig notwendigen - stationären Wiedereinweisung einen ganz wesentlichen „Vorteil“ dar.

Frage: Wie bewerten Sie speziell die Ergebnisse in CHARM-Added?

Prof. Krönig: Beeindruckend ist auch, dass trotz eines in der Regel schon sehr ausgereizten therapeutischen Prinzips, die Zugabe des $\mathrm{AT}_{1}$ Rezeptorblockers, hier am Beispiel von Candesartan, in der Lage war, die kardiovaskuläre Mortalität noch weiter zu reduzieren und gleichzeitig eine gute Verträglichkeit dieser umfangreichen Therapie sicherzustellen. Häufig wird eine wirksame zusätzliche Therapiekomponente bei schwierig zu behandelnden Krankheitsbildern durch eine schlechtere Verträglichkeit erkauft.

Frage: Haben Sie diese Ergebnisse schon in Ihrer therapeutischen Praxis umgesetzt?

Prof. Krönig: Nachdem im klinischen Bereich häufiger Patienten mit sehr schwerer Herzinsuffizienz $\mathrm{zu}$ finden sind, ist es eine erfreuliche
Erweiterung der Behandlungsmöglichkeiten gewesen, neben der „Basistherapie“ bei speziellen Patienten den $\mathrm{AT}_{1}$-Rezeptorblocker Candesartan einsetzen zu können. Dies gilt zum Beispiel für jene Patienten, die trotz guter Compliance immer wieder mit akuter Luftnot und verstärkten Ödemen zur Aufnahme kommen. Bisher haben wir gute Erfahrungen bei 20 bis 30 Patienten meist multimorbiden Patienten mit gleichzeitig schwer einstellbarer Hypertonie und einem Diabetes mellitus - sammeln können. Die beiden letztgenannten Indikationen sind darüber hinaus ebenfalls gut mit dem $\mathrm{AT}_{1}$-Rezeptorblocker anzugehen.

Frage: Was waren Ihre speziellen Beweggründe für diese Entscheidung?

Prof. Krönig: Auch in der Klinik, wie im Bereich der niedergelassenen Kollegen, ist es uns ein wichtiges Anliegen, Patienten mit Herzinsuffizienz möglichst rasch und effektiv beschwerdefrei, beziehungsweise zumindest deutlich beschwerdegelindert zu bekommen. Das war sicher der entscheidende Moment, um die Therapie zu erweitern, denn es ist schon eine ganz erhebliche Beeinträchtigung der Lebensqualität, wenn bei der Herzinsuffizienz, die ja nach wie vor ein sehr ernsthaftes Leiden darstellt, nur ein Teilerfolg erreicht wird. Selbst wenn die leit- 
liniengerechte Medikation in der Herzinsuffizienztherapie scheinbar ausgeschöpft ist, und wir eben nicht zu einer Verbesserung der Lebensqualität gekommen sind, müssen wir uns überlegen, ob wir nicht noch eine sinnvolle Erweiterung der Therapie vornehmen können. Das ist ja mit dem $\mathrm{AT}_{1}$-Rezeptorblocker Candesartan tatsächlich der Fall.

Die Ergebnisse sind aufgrund der Studienanlage als „evidence-basedmedicine“ anzusehen, wie dies heute von uns in der Therapie verlangt wird.

Frage: Wie gehen Sie praktisch vor, wenn Sie einen Patienten ergänzend zur Therapie mit ACE-Hemmern auch mit Candesartan therapieren?

Prof. Krönig: Natürlich müssen die Patienten selektiert werden. Es ist sicher nicht jeder Patient mit einer Herzinsuffizienz (NYHA II oder auch III) schon ein idealer Kandidat für die zusätzliche Therapie mit Candesartan. Hier sind es Patienten, die meist eine lange Vorgeschichte haben. Oft sind es solche, die auf dem Boden einer koronaren Herzerkrankung, aber auch einer fortgeschrittenen hypertensiven Herzkrankheit, über viele Jahre bereits mit vielen Substanzen behandelt worden sind und eben nicht aus ihrer Herzinsuffizienz ganz herausgekommen sind. Das sind die idealen und auch die dankbaren Patienten, die trotz einer Therapieerweiterung meist eine hohe Compliance an den Tag legen.

Nun wird man natürlich fragen, wie sieht es mit der Dosierung eben dieser $\mathrm{AT}_{1}$-Rezeptorblocker in der Therapie aus. Hier ist es sicher so, dass man nicht zu niedrig einsteigen kann und darf, denn diese Patienten sind ausgesprochen schwer erkrankt. Wir beginnen in der Regel bereits mit $16 \mathrm{mg}$ Candesartan und versuchen dann, eventuell bis auf $32 \mathrm{mg}$ täglich zu steigern. Aber das sind dann schon ganz spezielle Patienten, bei denen wir diese Dosissteigerung in einem Zeitraum von zwei bis vier Wochen erreichen und nicht ad hoc.

Erstaunlich ist auch, dass selbst Patienten die nicht hypertensiv sind, sondern eher im normotensiven Be- reich liegen, diese zusätzliche Substanz ausgesprochen gut tolerieren. Man könnte spekulieren, dass dies mit einer Verbesserung der endothelialen Dysfunktion und der Mikrozirkulation zusammenhängt, sodass auch zerebrale oder zerebrovaskuläre Vorschädigungen nicht so zum Tragen kommen, wie das unter Umständen bei anderen Substanzen, die den Druck senken, der Fall sein kann.

Zielkriterium der Therapie ist die weitestgehende Beschwerdefreiheit unserer Patienten. Wir müssen uns im Klaren darüber sein, dass man bei diesen Patienten nur eine bis zu einem gewissen Grad vollkommene Beschwerdefreiheit erreichen kann. Aber ich denke, dass jemand, der über Jahre hinweg schwer herzinsuffizient war und durch die Erweiterung der Therapie wieder in die Lage versetzt wird, am sozialen Leben teilzunehmen, anstatt nur einen Aktionsradius vom Sessel zum Sofa und umgekehrt $\mathrm{zu}$ haben, einen enormem Zugewinn seiner Lebensqualität erfährt.

Frage: Man könnte ja auch anmerken, dass Sie durch die zusätzliche Gabe von Candesartan bei Ihrer Therapie der Herzinsuffizienz die Kosten erhöhen. Wie begründen Sie Ihre Therapieentscheidung aus diesem Blickwinkel?

Prof. Krönig: Es ist natürlich vollkommen klar, dass die wiederholte Krankenhauseinweisung bei schwerer Herzinsuffizienz eine erhebliche Kostenbelastung des Gesundheitssystems darstellt, und dass jede Maßnahme, die zu einer Begrenzung der Krankenhauseinweisungen und auch der Dauer der Krankenhausbehandlung mit sich bringt, enorm viele Kosten einsparen hilft. Wir können im Durchschnitt davon ausgehen, dass die Pflegesätze in den internistischen Krankenhausabteilungen um die 300 bis 350 Euro pro Tag liegen. Wenn wir CandesartanDosen von 16 bis maximal $32 \mathrm{mg}$ gegenüberstellen, so sind das etwa nur 0,3\% der Kosten eines Krankenhaustages.

Es gibt aber noch einen weiteren Punkt, nämlich die signifikante Reduktion des Auftretens neuer Typ 2-
Diabetes-Patienten um 22\% durch Candesartan. Jeder weiß, wie hoch die Folgekosten des Diabetes sind.

Frage: Welche Empfehlungen geben Sie Ihren Kollegen für die Therapie der Herzinsuffizienz, die noch nicht zusätzlich mit Candesartan therapieren?

Prof. Krönig: Es wird bei allen Patienten immer zunächst die übliche Therapie, die auch die Allgemeinmaßnahmen umfasst, auszuschöpfen sein, aber man sollte eben sehr genau hinsehen, inwieweit die Patienten wirklich beschwerdefrei geworden sind. Dabei ist es schon erstaunlich, dass eine Reihe von Patienten, die eine Therapie aus ACEHemmern, Diuretika/Spironolacton und gegebenenfalls Betablockern, durchführen, trotzdem noch eine unzureichende Beeinflussung ihrer Herzinsuffizienz erleben. Das sind genau jene Patienten, bei denen die zusätzliche Gabe eines $\mathrm{AT}_{1}$-Rezeptorblockers, wie eben von Candesartan, indiziert sein kann.

Diese prinzipiell wichtige Bereicherung unseres Therapiekonzeptes, wie es durch die CHARM-Studie belegt worden ist, muss allerdings noch stärker ins Bewusstsein unserer Kollegenschaft in Klinik und Praxis gebracht werden. Dabei spielt sicher auch die persönliche Erfahrung eines jeden Kollegen eine wichtige Rolle. Wie mit allen neuen, beziehungsweise neueren Therapieprinzipien wird man sehen müssen, wie sich bei Patienten mit fortgeschrittener Herzinsuffizienz eben nicht nur das Befinden, sondern auch die übrigen messbaren Parameter bessern lassen.

\section{Herr Professor Krönig, wir be- danken uns für dieses Gespräch!}

\author{
Korrespondenzadresse \\ Prof. Dr. med. Bernd Krönig \\ Arzt für Innere Medizin \\ Chefarzt der Inneren Abteilung \\ des Ev.Elisabeth-Krankenhauses \\ Theobaldstr. 12 \\ 54292 Trier \\ Fax: 0651/2092-290 \\ E-Mail: BKroenig@aol.com
}

